\title{
Delays in processing and storage of pig seminal plasma alters levels of contained antioxidants
}

Isabel Barranco, Asta Tvarijonaviciute, Lorena Padilla, Heriberto RodriguezMartinez, Jordi Roca and Xiomara Lucas

The self-archived postprint version of this journal article is available at Linköping University Institutional Repository (DiVA):

http://urn.kb.se/resolve?urn=urn:nbn:se:liu:diva-175294

N.B.: When citing this work, cite the original publication.

Barranco, I., Tvarijonaviciute, A., Padilla, L., Rodriguez-Martinez, H., Roca, J., Lucas, X., (2021), Delays in processing and storage of pig seminal plasma alters levels of contained antioxidants, Research in Veterinary Science, 135, 416-423. https://doi.org/10.1016/j.rvsc.2020.10.027

Original publication available at:

https://doi.org/10.1016/j.rvsc.2020.10.027

Copyright: Elsevier

http://www.elsevier.com/

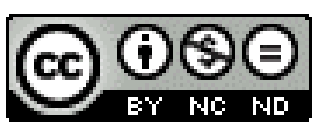




\title{
Delays in processing and storage of pig seminal plasma alters levels of contained antioxidants
}

\author{
Isabel Barranco $^{\mathrm{a}, \mathrm{b}}$, Asta Tvarijonaviciute ${ }^{\mathrm{a}}$, Lorena Padilla ${ }^{\mathrm{a}}$, Inmaculada Parrilla ${ }^{\mathrm{a}}$, \\ Heriberto Rodriguez-Martinez ${ }^{\mathrm{c}}$, Jordi Roca ${ }^{\mathrm{a}, *}$, Xiomara Lucas $^{\mathrm{a}}$
}
${ }^{\text {a }}$ Department of Medicine and Animal Surgery, Faculty of Veterinary Science, University of Murcia, Murcia, 30100, Spain
${ }^{b}$ Department of Biology, Faculty of Sciences, Institute of Food and Agricultural Technology, University of Girona, Girona, 17003, Spain
${ }^{\mathrm{c}}$ Department of Biomedical and Clinical Sciences (BKV), Linköping University, Linköping, SE-58185, Sweden

*Corresponding author: roca@um.es (Jordi Roca)

Department of Medicine and Animal Surgery, Veterinary Teaching Hospital 3 Floor, University of Murcia, Campus Espinardo, Murcia, 30100 (Spain) 


\begin{abstract}
Seminal plasma (SP) antioxidants are considered biomarkers of sperm function and fertility for AI-boars. The current protocol for their measurement implies the SP was harvested immediately after ejaculation and prompt stored at $-80^{\circ} \mathrm{C}$ until analysis. Such protocol may be impractical for AI-centers. This study evaluated how SP levels of antioxidants were influenced by delays in (1) SP-harvesting ( 0 [control], 2 or $24 \mathrm{~h}$ at $17^{\circ} \mathrm{C}$ after ejaculate collection), in (2) SP-freezing (0 [control] or $24 \mathrm{~h}$ at $17^{\circ} \mathrm{C}$ after SP-harvesting) or (3) the temperature of storage $\left(-80^{\circ} \mathrm{C}\right.$ [control] or $\left.-20^{\circ} \mathrm{C}\right)$. The SP-antioxidants evaluated were: glutathione peroxidase [GPx], superoxide dismutase [SOD], paraoxonase-1 [PON-1], trolox equivalent antioxidant capacity [TEAC] and oxidative stress index [OSI]. A total of 120 aliquots from 10 entire ejaculates were handled in three trials. They were centrifuged (1,500g, $10 \mathrm{~min})$ for harvesting SP and antioxidants were measured with an Automatic Chemistry Analyzer. A 24h-delay in harvesting the SP led to an increase $(p<0.001)$ in TEAC and SOD SP-levels, and a decrease $(p<0.05)$ of OSI and PON-1. Similarly, a 24h-delay to freeze the SP increased $(p<0.01)$ TEAC values and decreased $(p<0.01)$ PON-1 and GPx activity levels. Finally, storing the SP at $-20^{\circ} \mathrm{C}$ decreased $(p<0.001)$ SP-levels of TEAC, PON-1 and GPx, and increased $(p<0.01)$ OSI values. Strong positive relationships $(p<0.001)$ were found between antioxidant SP-levels in processed samples and their respective controls. In sum, handling and SP storage influence antioxidant measurements in AI-boars. Reliable levels of SP-antioxidants can only be warranted if a strict protocol for harvesting and SP storage is followed.
\end{abstract}

Keywords: antioxidants, pig, seminal plasma, storage, processing

\title{
1. Introduction
}


In the swine industry, artificial insemination (AI) is considered an indispensable tool for its efficiency to increase productivity and genetic progress (Roca et al., 2016a; Waberski et al., 2019). Recent improvements in AI-procedures leading to a reduction in both sperm numbers per AI-dose and the number of AI-doses per inseminated sow without impairing fertility outcomes, sharpens the level of influence that AI-boars have on the reproductive and hence the economic outcomes of breeding farms (Roca et al., 2016a). Therefore, predicting the fertilizing capacity of AI-boars is utterly important. Currently, conventional semen evaluation, including sperm quantity, motility and morphology, are used to class boar ejaculates for AI (Broekhuijse et al., 2011; Roca et al., 2016a; Waverski et al., 2019). However, the clear and evident differences between AI-boars in the ability of their sperm to sustain preservation, and in fertility post-AI, demands searching for better biomarkers (Parrilla et al., 2012; Roca et al., 2015). Consequently, current research has explored eventual biomarkers in seminal plasma (SP), a complex fluid mainly secreted by accessory sexual glands, that interacts with spermatozoa and the female genital tract once delivered in during insemination (Rodriguez-Martinez et al., 2011).

Seminal plasma is rich in antioxidants protecting sperm against the oxidative stress (Barranco et al., 2015a) which is particularly damaging to boar spermatozoa due to its peculiar lipid plasma membrane composition (Strzezek et al., 1999; Bathgate, 2011; Koziorowska-Gilun et al., 2011). Recent studies have demonstrated the relevance of SPantioxidants, specifically superoxide dismutase (SOD), glutathione peroxidase (GPx), paraoxonase 1 (PON-1) and the trolox equivalent antioxidant capacity (TEAC), for boar sperm function, preservation and fertility after AI (Barranco et al., 2015a, 2015b, 2016, 2019a; Li et al., 2018). Consequently, measurement of the levels of these antioxidants in SP 
has been suggested as complementary tests to select boars for best semen production (Barranco et al., 2015a, 2015b, 2016, 2019a).

The optimal protocol used in the above studies to measure the SP-antioxidants included harvesting the SP immediately after ejaculation, to reduce the potential interference with sperm, and storage at $-80^{\circ} \mathrm{C}$, to avoid any degradation. The SP was stored for up to $12-$ months until analyzed in central laboratories, which were generally located away from AIcenters. Indeed, a similar SP processing protocol that involves immediate SP harvesting after ejaculate collection and their storage at $-80^{\circ} \mathrm{C}$ or $-70^{\circ} \mathrm{C}$, has been widely used in human studies for reliable SP antioxidant measurements (Peeker et al., 1997; Mahfouz et al. 2009, Benedetti et al., 2012; Otasevic et al., 2019). This handling proved useful for research with pigs, but is often unpractical in many AI-centers which, mainly for technical and working routine reasons claim for alternative protocols. Accordingly, the present study aimed to investigate the effects of (1) delaying SP-harvesting after ejaculate collection; (2) delaying the freezing of the harvested SP; and (3) the suitability of SP storage at $-20{ }^{\circ} \mathrm{C}$, for their effects in the measurements of relevant SP-antioxidants, specifically GPx, SOD, PON-1, TEAC and Oxidative Stress Index (OSI).

\section{Material and methods}

\subsection{Ethics statement}

The semen samples used in the experiment were provided by the AI-center of AIM Iberica (Topigs Norsvin Spain SLU) located in Calasparra (Spain). The center fulfills both Spanish (ES300130640127, August 2006) and European (ES13RS04P, July 2012) standards 
for animal health and welfare and it is authorized for collection and commercialization of boar semen.

\subsection{Boars, ejaculates and seminal plasma}

Entire ejaculates were collected from mature (1.5 to 3 years), healthy and fertile AIboars (Landrace, Pietrain and Tempo) using a semi-automatic method (Collectis ${ }^{\circledR}$, IMV Technologies, L'Aigle, France). Boars were housed in individual stalls in a closed building with controlled environmental conditions ( $16 \mathrm{~h}$ of daylight and $24-25^{\circ} \mathrm{C}$ of air temperature). The boars were subjected to regular semen collection of two ejaculates per week for the preparation of commercial AI-semen doses. The ejaculates used in this study fulfilled the standards of quantity and sperm quality mandatory for liquid AI-semen doses, namely more than $200 \times 10^{6}$ sperm $/ \mathrm{mL}$ with $70 \%$ and $75 \%$ of them showing motility and normal morphology, respectively. All boars were free of chromosomal translocations and displayed rates of sperm nuclear chromatin fragmentation below $3 \%$.

Seminal plasma was harvested by double centrifugation at $1,500 \mathrm{~g}$ at room temperature (RT) for 10 min (Rotofix 32A; Hettich Centrifuge UK, Newport Pagnell, Buckinghamshire, England, UK). The resulting SP samples were examined microscopically (Eclipse E400; Nikon, Tokyo, Japan) to ensure the total absence of spermatozoa. Subsequently, each SP sample was individually stored in cryotubes $(3 \mathrm{~mL})$ and processed as indicated in the experimental design (see below, Fig 1).

\subsection{Measurement of seminal plasma antioxidants}

The TEAC, OSI and the activity of GPx, PON-1 and SOD were measured in SP samples using an automated biochemistry analyzer (Olympus AU600 Automatic Chemistry 
Analyzer, Olympus Europe GmbH, Germany). Each assay exhibited an intra- and inter-assay coefficient variations below $10 \%$, depicting high linearity under serial dilutions. Measurements were made in duplicate for each SP-sample.

\subsubsection{Trolox equivalent antioxidant capacity (TEAC)}

The TEAC was assessed using a protocol previously described by Erel (2004). The method was based on 2,2' -azinobis-(3-ethylbenzothiazoline-6-sulfonate) acid radical cation decolorization by non-enzymatic antioxidants, in accordance with their concentrations and antioxidant capacities. The color modification was assessed by changes in light absorbance

at $660 \mathrm{~nm}$. This assay was calibrated by 6-hydroxy-2, 5, 7, 8-tetramethylchroman-2carboxylic acid (Trolox), expressing the TEAC-value of each SP sample as an equivalent of the mM-concentration of Trolox solution (mmol Trolox equiv./L).

\subsubsection{Total glutathione peroxidase $(G P X)$ activity}

The total GPX activity was measured using a commercially available kit (RANSEL kit, RANDOX Laboratories, Crumlin, United Kingdom), following the manufacturer guidelines. Briefly, GPx catalyzes the oxidation of glutathione using cumene hydroperoxide and when NADPH and glutathione reductase were present, the oxidized glutathione was converted to the reduced form with immediate oxidation of NADPH to NAD+. The decrease in light absorbance was measured at $340 \mathrm{~nm}$ and the total GPx activity was defined as one unit to oxidize one $\mu \mathrm{mol}$ NADPH in one min, expressed as IU/L.

\subsubsection{Paraoxonase 1 (PON-1) activity}


The PON-1 enzymatic activity was measured following the procedure described by Barranco et al. (2015b). Briefly, PON-1 activity was measured in vitro, assessing the hydrolysis of p-nitrophenyl acetate to p-nitrophenol. Before this analysis, acetazolamide $(0.5$ $\mathrm{mM}$ ) and di-isopropyl fluorophosphates $(0.5 \mathrm{mM})$ were added to the SP samples, in order to warrant that there were no contaminant enzymes able to hydrolyze p-nitrophenyl acetate. The PON-1 activity was expressed as IU/mL.

\subsubsection{Superoxide dismutase (SOD) activity}

The SOD activity was assessed using a commercially available kit (RANSOD kit, RANDOX Laboratories), following the manufacturer guidelines. Briefly, this method was based on the formation of red formazan dye, generated by the reaction between the superoxide radical (produced by xanthine and xanthine oxidase) with 2-(4-iodophenyl)-3-(4nitrophenol)-5-phenyltetrazolium chloride (INT). Then, the SOD activity was assessed as the degree of inhibition of this reaction (one unit of SOD corresponds to a 50\% inhibition of the rate of reduction of INT). The color change was evaluated by changes in light absorbance at $505 \mathrm{~nm}$ and the SOD activity was expressed as IU/mL.

\subsubsection{Oxidative stress index (OSI) determination}

The total oxidative status (TOS) of SP was measured following a previously described procedure $(\mathrm{Erel}, 2005)$. This method was based on the oxidation $\mathrm{Fe}^{2+}$ to $\mathrm{Fe}^{3+}$ in acidic medium and in the subsequent interaction between $\mathrm{Fe}^{3+}$ with xylenol orange. This reaction gives a colored complex, whose intensity was consistent with the total of oxidants present in the SP sample. This assay was calibrated using $\mathrm{H}_{2} \mathrm{O}_{2}$ and the color change was evaluated by changes in light absorbance at $560 \mathrm{~nm}$. The TOS was expressed as $\mu \mathrm{mol} \mathrm{H}_{2} \mathrm{O}_{2}$ 
equiv./L. The OSI value was calculated according to the following formula (Wu et al., 2017): OSI (arbitrary unit) $=$ TOS $\left(\mu \mathrm{mol} \mathrm{H}_{2} \mathrm{O}_{2}\right.$ equiv./L)/TEAC (mmol Trolox equiv./L).

\subsection{Experimental design}

The experiment used ten ejaculates (one per boar) in a factorial study $3 \times 2 \times 2$ (Fig. 1) to evaluate (1) three lapsed times between collection of ejaculate and harvesting of SP (0 [control], 2 and 24 h); (2) two elapsed times from SP harvest to freezing (0 [control] and 24 h); and (3) two frozen temperatures for storage $\left(-80^{\circ} \mathrm{C}\right.$ [control] and $\left.-20^{\circ} \mathrm{C}\right)$. A total of 12 SP samples were obtained from each of the ten ejaculates. All SP samples were stored frozen for 12 months period in the Laboratory of the Veterinary Teaching Hospital of the University of Murcia (Spain) before antioxidant measurements on RT-thawed SP. Instability of each antioxidant was assessed comparing the measurements in SP samples obtained under different processing and storage conditions with those found in its respective SP samples obtained and stored frozen at $-80{ }^{\circ} \mathrm{C}$ immediately after ejaculate collection (control).

As shown in Fig. 1, semen samples of the ten ejaculates were split into three aliquots that were twice centrifuged either immediately after ejaculate collection or after 2 and $24 \mathrm{~h}$ of storage at $17{ }^{\circ} \mathrm{C}$. Each resulting SP-sample was split in two aliquots and one was immediately frozen (a) whereas the other (b) remained stored at $17^{\circ} \mathrm{C}$ for $24 \mathrm{~h}$ before being frozen. Immediately prior to its frozen storage, these SP samples were again split into two aliquots: (x) one aliquot was stored at $-20^{\circ} \mathrm{C}$ (Zanussi Tropic System, Electrolux España S.A.U, Madrid, Spain) and (y) the other aliquot at $-80^{\circ} \mathrm{C}$ (control; Ultra-Low Freezer; Haier Inc., Qingdao, China).

\subsection{Statistical analysis}


The IBM SPSS Statistics package version 24.0 (IBM Spain, Madrid) was used for the statistical analysis. Firstly, Shapiro-Wilk test was performed for testing the assumption of normality and data that were not normally distributed were square root transformed. A mixed ANOVA model and Bonferroni post-hoc test were used for evaluating the effects of elapsed time for SP harvesting (0 [control], 2 and $24 \mathrm{~h}$ ), elapsed time from SP harvest to frozen storage (0 [control] and $24 \mathrm{~h})$; and two freezing storage conditions for SP samples $\left(-80^{\circ} \mathrm{C}\right.$ [control] or $-20{ }^{\circ} \mathrm{C}$ ) on SP antioxidant measurements, considering ejaculates as random effect. The mixed ANOVA analysis revealed no interactions $(p>0.05)$ between the processing and storage conditions evaluated. Therefore, new mixed ANOVAs were performed for evaluating the effect of each one of processing and storage condition, including the two other conditions and ejaculates as random effects. Pearson's correlation coefficient test was applied to assess the relationship between SP antioxidant levels measured in the different processing and storage conditions. A p-value $<0.05$ was considered to be statistically significant.

\section{Results}

The mixed ANOVA analysis revealed no interactions $(p>0.05)$ between the processing conditions evaluated for semen and SP. Therefore, the effect of each condition on antioxidant measurements was separately evaluated. Looking at the first one, the delay in SP harvesting after ejaculate collection increased $(p<0.001)$ TEAC and SOD SP-levels, and decreased $(p<0.05)$ OSI and PON-1 (Fig. 2, Supplementary Table S1). However, while the $24 \mathrm{~h}$ delay either increased or decreased the SP measurements of the four antioxidants, the 2 h delay only increased $(p<0.001)$ those of TEAC and SOD with the delay as time-length. In contrast, OSI values and PON-1 activity levels were lower $(p<0.05)$ in SP samples 
harvested $24 \mathrm{~h}$ after ejaculate collection than in those harvested $2 \mathrm{~h}$ or immediately after ejaculate collection. For all antioxidants assessed, their levels in SP samples harvested immediately after ejaculate collection (control) were positively correlated $(p<0.001$; coefficients $[\mathrm{r}]>0.75)$ with those found in SP samples harvested 2 and $24 \mathrm{~h}$ after ejaculate collection (Fig. 3).

Regarding the second processing condition evaluated, a delay of $24 \mathrm{~h}$ in the freezing of SP increased $(p<0.01)$ the TEAC levels and decreased $(\mathrm{p}<0.01)$ PON-1 and GPx values (Fig. 4, Supplementary Table S2). The PON-1 and GPx activity levels were lower $(p<0.01)$ and the TEAC levels higher $(p<0.01)$ in the SP samples frozen $24 \mathrm{~h}$ after SP harvesting than in those immediately frozen. For all antioxidants, their levels in SP frozen immediately after the SP was harvested ( $0 \mathrm{~h}$; control) were positively correlated $(p<0.001 ; \mathrm{r}>0.9)$ with those found in SP samples frozen $24 \mathrm{~h}$ after SP harvest (Fig. 5).

Finally, regarding the third SP processing condition evaluated, storage of SP samples at $-20{ }^{\circ} \mathrm{C}$ decreased $(p<0.001)$ SP-levels of TEAC, PON-1 and GPx, and increased $(p<$ 0.01) OSI values (Fig. 6, Supplementary Table S3). The activity levels of TEAC, PON-1 and GPx were higher $(p<0.001)$ in the SP samples stored at $-80{ }^{\circ} \mathrm{C}$ than in those stored at -20 ${ }^{\circ} \mathrm{C}$. In contrast, OSI values were lower $(p<0.01)$ in the SP samples stored at $-80{ }^{\circ} \mathrm{C}$ than in those stored at $-20{ }^{\circ} \mathrm{C}$. For all antioxidants, their levels in SP samples frozen at $-80{ }^{\circ} \mathrm{C}$ (control) were positively correlated $(\mathrm{p}<0.001 ; \mathrm{r}>0.75)$ with those found in SP samples frozen at $-20^{\circ} \mathrm{C}$ (Fig. 7).

\section{Discussion}

To the best of our knowledge, this is the first report in livestock evaluating the effects of SP-processing conditions on the stability of some antioxidants measured in SP samples. 
The results evidenced that the processing conditions tested affected to a greater or lesser extent the five antioxidants analyzed, namely TEAC, OSI, PON-1, SOD and GPx.

Harvesting SP immediately after ejaculate collection is sometimes a difficult task to adapt to the routine work of the AI-centers since it requires time and extra labor. Under this scenario, our first goal was to evaluate whether the delay in SP harvesting would affect the SP measurement of antioxidants. Delaying SP harvest would also allow sending raw semen samples to central laboratories to harvest and SP analysis. To simulate a delay in SP harvesting, freshly semen samples were stored for 2 and $24 \mathrm{~h}$ at $17^{\circ} \mathrm{C}$, the usual temperature used in pig AI-centers for storing semen AI-doses. A delay of $2 \mathrm{~h}$ would allow the SP harvest to be adapted to the routine work of the center and a delay of up to $24 \mathrm{~h}$ would allow the raw semen to be sent to the central laboratories. The results demonstrated that a delay of $24 \mathrm{~h}$ in harvesting the SP should not be applied since it causes quantitative changes in many of the measured SP antioxidants, specifically for TEAC, OSI, PON-1 and SOD. A delay of maximum $2 \mathrm{~h}$ would be more practical but it also causes quantitative changes in two of the five SP antioxidant measured. The GPx was the only antioxidant measured whose SP activity values were not affected by the delays in SP harvesting hereby tested. Although no similar studies have been performed in SP, the effect of delaying centrifugation in blood affecting serum or plasma analytes, has been extensively reported in human (Clark et al., 2003; Karlsen et al., 2007; Tanner et al., 2008; Oddoze et al., 2012; Henriksen et al., 2014; Abraham et al., 2019). In agreement with our results, these studies revealed that some analytes (including enzymatic and non-enzymatic antioxidants) measured in blood serum or plasma were affected by time (from $4 \mathrm{~h}$ to $24 \mathrm{~h}$ ) and a variety of temperatures (from $2{ }^{\circ} \mathrm{C}$ to $35^{\circ} \mathrm{C}$ ) during which the whole blood was kept before centrifugation (Karlsen et al., 2007; Tanner et al., 2008; Henriksen et al., 2014; Abraham et al., 2019). Abraham et al. (2019) postulated that 
the continuous exchange between serum and cells along with loss of membrane integrity experienced by many cells in unprocessed blood samples leads to a constant release of intracellular content, increasing serum levels of many analytes. This transfer from cells, in the case of semen from spermatozoa to the surrounding SP, would explain the progressive increase in TEAC and SOD activity levels as the delay in SP-harvesting increased. The fact that tocopherol (one of the contributors of the TEAC assay) and SOD are present in boar spermatozoa (Breininger et al., 2011; Zura Zaja et al., 2016) and the progressive loss of membrane integrity experienced by spermatozoa once ejaculated (Roca et al., 2016b) would support the above statement. In contrast to SOD, the levels of PON-1 activity were lower in the SP samples harvested $24 \mathrm{~h}$ after ejaculate collection. The well-known progressive increase in reactive oxygen species (ROS) experienced by boar semen samples during storage at $17{ }^{\circ} \mathrm{C}$, leading to an increase in sperm membrane lipid peroxidation (Kumaresan et al., 2009; Barranco et al., 2019b), would be a rational explanation for the decreased levels in PON-1 observed in SP samples recovered later. Certainly, increased levels of oxidized lipids have been shown to inhibit PON-1 activity (Aviram et al., 1999). As expected, an increase in TEAC led to a decrease in OSI values, since TEAC is the denominator in the formula for determining OSI.

Next, whether the harvested SP could be kept chilled for $24 \mathrm{~h}$ before frozen for storage was examined. This procedure would facilitate the handling of SP samples in AIcenters since they have refrigerated chambers but rarely $-80{ }^{\circ} \mathrm{C}$ freezers. Therefore, a delay in the freezing of SP samples was experimentally simulated by preserving them for $24 \mathrm{~h}$ at $17^{\circ} \mathrm{C}$, an interval and temperature classically used to send extended semen (or SP samples) from the AI-centers to the farms (or central laboratories). The results demonstrated that a delay of $24 \mathrm{~h}$ in freezing the SP decreased the levels of PON-1 and GPx and increased TEAC 
levels, but did not affect SOD or OSI. Low-temperature causes inhibition or slowing of enzyme action and thus the rates of chemical reactions they catalyzed (Georlette et al., 2004). This may explain the lower activity levels for PON-1 and GPx observed in the SP stored at $17^{\circ} \mathrm{C}$ for $24 \mathrm{~h}$ before freezing. Another putative explanation for the lower GPx activity would be a decrease in the availability of glutathione, the substrate, whose levels were found to decrease in human milk stored at $4{ }^{\circ} \mathrm{C}$ or at RT for $2 \mathrm{~h}$ (Ankrah et al., 2000). More difficult to understand was the lower activity of PON-1 in the samples cooled for $24 \mathrm{~h}$, an enzyme considered stable in body fluids when preserved by refrigeration, as it has been demonstrated with saliva (Rossi et al., 2013; Rubio et al., 2018). The stability of antioxidant measurements has been extensively evaluated in other biological fluids (e.g. blood serum, saliva or milk) of different mammalian species, including pigs, stored at $4{ }^{\circ} \mathrm{C}$ and/or at $\mathrm{RT}\left(25^{\circ} \mathrm{C}\right)$ for at least 24 h, achieving contradictory results (Craft et al., 1988; Hooser et al., 2000; Hanna et al., 2004; Celi et al., 2010; Rossi et al., 2013; Rubio et al., 2018; Barranco T. et al., 2019). For instance, Rubio et al. (2018) reported that measurements of antioxidant status, including $\mathrm{TEAC}_{\mathrm{H}}$, in dog saliva samples were affected by storage of the samples for $24 \mathrm{~h}$ at $4{ }^{\circ} \mathrm{C}$ or 25 ${ }^{\circ} \mathrm{C}$. However, the same antioxidant status measurements were not affected in human milk and saliva samples stored for 48 and $72 \mathrm{~h}$ at $4{ }^{\circ} \mathrm{C}$, respectively (Hanna et al., 2004; Barranco T. et al., 2019). In pigs, Hooser et al. (2000) reported that tocopherol concentrations (one of the contributors of the TEAC assay) remained stable in serum samples stored for 14 days at $4{ }^{\circ} \mathrm{C}$. Taken together, these results would indicate that the stability in the measurement of antioxidants in cool-stored samples would depend on the antioxidant, biological fluid- and the species considered.

The last objective of this study was to assess whether sub-zero storage temperatures affected the levels of antioxidants in pig SP. For this, the suitability of using freezers of -20 
${ }^{\circ} \mathrm{C}$ was tested, since they are more affordable for AI-centers than those at $-80{ }^{\circ} \mathrm{C}$. The results showed clear differences in the levels of four of the five antioxidants measured in SP stored at $-20^{\circ} \mathrm{C}$ or $-80^{\circ} \mathrm{C}$. The SOD activity was the only unaffected. The effect of sub-zero storage on antioxidant levels has been extensively reported for several biological fluids other than SP, primarily humans and laboratory species (Craft et al., 1988; Jansen et al., 2013; Bortolin et al., 2017; Rubio et al., 2018; Barranco T. et al., 2019). Bortolin et al., (2016) reported in rat blood that the effects ultimately depend on the specific antioxidant analyzed, as our results also revealed. Alike our results, the study of Bortolin et al. (2016) showed similar SOD activity levels between blood samples stored at $-20^{\circ} \mathrm{C}$ and at $-80^{\circ} \mathrm{C}$ for 2 months. However, it also showed that catalase activity levels (another antioxidant enzyme) in blood samples stored at $-20^{\circ} \mathrm{C}$ were lower than in those stored at $-80{ }^{\circ} \mathrm{C}$. Similarly, and also in agreement with our results, Rubio et al. (2018) showed lower PON-1 activity levels in dog saliva samples stored at $-20^{\circ} \mathrm{C}$ compared with those stored at $-80^{\circ} \mathrm{C}$ for 12 months. The lower GPx activity levels in SP samples stored at $-20{ }^{\circ} \mathrm{C}$ with respect to those stored at $-80{ }^{\circ} \mathrm{C}$ could also be related to the substrate availability, as was the case for human milk (Ankrah et al., 2000). In relation to TEAC, the lowest activity levels in SP stored at $-20{ }^{\circ} \mathrm{C}$ was also found in $\operatorname{dog}$ saliva and human serum (Jansen et al., 2013; Rubio et al., 2018). The better stability of biomolecules involved in TEAC assay, as carotenoids and tocopherol, at $-80{ }^{\circ} \mathrm{C}$ or $-70{ }^{\circ} \mathrm{C}$ than at $-20^{\circ} \mathrm{C}$ would be key to higher levels of TEAC measured in samples stored at - 80 or $70^{\circ} \mathrm{C}$ than in those stored at $-20^{\circ} \mathrm{C}$ (Mathews-Roth and Stampfer, 1984; Craft et al., 1988).

Finally, it is important to highlight the strong positive relationships found between the levels of antioxidants in SP processed under the optimal protocol with those processed and stored under alternative conditions. Key et al. (1996) also reported strong positive correlations in human serum between the concentration of carotenoids, acid ascorbic and 
tocopherol (contributors for TEAC assay) in blood stored at $4{ }^{\circ} \mathrm{C}$ for 2 with those stored for 6 and $24 \mathrm{~h}$ prior to serum harvest. Similarly, Jansen et al. (2013) reported a strong positive correlation between TEAC values measured in human serum stored at $-20{ }^{\circ} \mathrm{C}$ and at $-80{ }^{\circ} \mathrm{C}$. Although these strong positive relationships found in all SP-antioxidants measured would suggest that any processing and storage conditions could be useful, the clear differences in antioxidant levels encountered oblige us to demand caution when used for SP-antioxidant assessments in AI-boars.

\section{Conclusions}

Optimal measurements of antioxidant levels in pig SP samples demand the SP to be harvested immediately after ejaculate collection and immediately stored at $-80{ }^{\circ} \mathrm{C}$ until analysis. However, focusing on individual antioxidant measurements: (1) OSI, GPx and PON-1 activity could be alternatively measured in SP harvested in semen samples stored at $17^{\circ} \mathrm{C}$ for $2 \mathrm{~h}$ (PON-1 and OSI) or for $24 \mathrm{~h}$ (GPx) after ejaculate collection; (2) OSI and SOD activity levels remain stable in SP samples stored at $17^{\circ} \mathrm{C}$ for $24 \mathrm{~h}$ prior freezing; and (3) SOD activity levels can be measured in SP samples stored at $-20^{\circ} \mathrm{C}$. The positive relationship found between the antioxidant levels measured in the different handled SP-samples, allows to design different practical protocols for harvesting and SP storage for each particular AIcenter/laboratory. However, protocols must be strict for reliable antioxidant measurements.

\section{Funding}

The study was funded by MINECO (Spain) and FEDER funds (EU) (AGL201569738-R), and Seneca Foundation Murcia, Spain (19892/GERM-15); The Swedish Research Council FORMAS (2017-00946 and 2019-00288), Stockholm, Sweden. L.P. was financially 
supported by MINECO (Madrid, Spain; BES-2016-076404). I.B and A.T were financially supported by MICINN (Madrid, Spain; FJCI-2017-31689 and RYC-2017-22992).

\section{Declaration of Competing Interest}

The authors have declared no conflict of interest.

\section{Acknowledgements}

The authors thank to AIM Iberica (Topigs Norsvin Iberica) for supplying the ejaculates.

\section{References}

Abraham, R.A., Agrawal, P.K., Acharya, R., Sarna, A., Ramesh, S., Johnston, R., de Wagt, A., Khan, N., Porwal, A., Kurundkar, S.B., Pandey, A., Pullakhandam, R., Nair, K.M., Kumar, G.T., Sachdev, H.P.S., Kapil, U., Saxena, R., Deb, S., Khera, A., Ramakrishnan, L., 2019. Effect of temperature and time delay in centrifugation on stability of select biomarkers of nutrition and non-communicable diseases in blood samples. Biochem. Medica 29, 20708.

Ankrah, N.A., Appiah-Opong, R., Dzokoto, C., 2000. Human breastmilk storage and the glutathione content. J. Trop. Pediatr. 46, 111-113.

Aviram, M., Rosenblat, M., Billecke, S., Erogul, J., Sorenson, R., Bisgaier, C.L., Newton, R.S., La Du, B., 1999. Human serum paraoxonase (PON 1) is inactivated by oxidized low density lipoprotein and preserved by antioxidants. Free Radic. Biol. Med. 26, 892-904. 
Barranco I., Tvarijonaviciute, A., Perez-Patiño, C., Parrilla, I., Ceron, J.J., Martinez, E.A., Rodriguez-Martinez, H., Roca, J., 2015a. High total antioxidant capacity of the porcine seminal plasma (SP-TAC) relates to sperm survival and fertility. Sci. Rep. 5, 18538.

Barranco, I., Tvarijonaviciute, A., Perez-Patino, C., Alkmin, D. V, Ceron, J.J., Martinez, E.A., Rodriguez-Martinez, H., Roca, J., 2015b. The activity of paraoxonase type 1 (PON-1) in boar seminal plasma and its relationship with sperm quality, functionality, and in vivo fertility. Andrology 3, 315-320.

Barranco, I., Tvarijonaviciute, A., Perez-Patino, C., Vicente-Carrillo, A., Parrilla, I., Ceron, J.J., Martinez, E.A., Rodriguez-Martinez, H., Roca, J., 2016. Glutathione peroxidase 5 is expressed by the entire pig male genital tract and once in the seminal plasma contributes to sperm survival and in vivo fertility. PLoS One 11, e0162958.

Barranco, I., Padilla, L., Tvarijonaviciute, A., Parrilla, I., Martínez, E.A., RodriguezMartinez, H., Yeste, M., Roca, J., 2019a. Levels of activity of superoxide dismutase in seminal plasma do not predict fertility of pig AI-semen doses. Theriogenology 140, 18-24.

Barranco, I., Padilla, L., Perez-Patino, C., Vazquez, J.M., Martinez, E.A., RodriguezMartinez, H., Roca, J., Parrilla, I., 2019b. Seminal Plasma Cytokines Are Predictive of the Outcome of Boar Sperm Preservation. Front. Vet. Sci. 6, 436.

Barranco, T., Rubio, C.P., Tvarijonaviciute, A., Rubio, M., Damia, E., Lamy, E., Cugat, R., Cerón, J.J., Tecles, F., Escribano, D., 2019. Changes of salivary biomarkers under different storage conditions: effects of temperature and length of storage. Biochem. Medica 29, 10706.

Bathgate, R., 2011. Antioxidant mechanisms and their benefit on post-thaw boar sperm quality. Reprod. Domest. Anim. 46 Suppl 2, 23-25. 
Benedetti, S., Tagliamonte, M.C., Catalani, S., Primiterra, M., Canestrari, F., De Stefani, S., Palini, S., Bulletti, C., 2012. Differences in blood and semen oxidative status in fertile and infertile men, and their relationship with sperm quality. Reprod. Biomed. Online $25,300-306$.

Bortolin, R.C., Gasparotto, J., Vargas, A.R., da Silva Morrone, M., Kunzler, A., Henkin, B.S., Chaves, P.R., Roncato, S., Gelain, D.P., Moreira, J.C.F., 2017. Effects of freeze-thaw and storage on enzymatic activities, protein oxidative damage, and immunocontent of the blood, liver, and brain of rats. Biopreserv. Biobank. 15, 182-190.

Breininger, E., Descalzo, A., Rossetti, L., Abramovich, D., Beconi, M.T., 2011. Boar sperm functionality is related to $\alpha$-tocopherol content after freezing-thawing. Andrologia 43, 409-415.

Broekhuijse, M.L.W.J., Feitsma, H., Gadella, B.M., 2011. Field data analysis of boar semen quality. Reprod. Domest. Anim. 46 Suppl 2, 59-63.

Celi, P., Sullivan, M., Evans, D., 2010. The stability of the reactive oxygen metabolites (d-ROMs) and biological antioxidant potential (BAP) tests on stored horse blood. Vet. J. 183, 217-218.

Clark, S., Youngman, L.D., Palmer, A., Parish, S., Peto, R., Collins, R., 2003. Stability of plasma analytes after delayed separation of whole blood: implications for epidemiological studies. Int. J. Epidemiol. 32, 125-130.

Craft, N.E., Brown, E.D., Smith, J.C.J., 1988. Effects of storage and handling conditions on concentrations of individual carotenoids, retinol, and tocopherol in plasma. Clin. Chem. 34, 44-48. 
Erel, O., 2004. A novel automated direct measurement method for total antioxidant capacity using a new generation, more stable ABTS radical cation. Clin. Biochem. 37, 277285.

Erel, O., 2005. A new automated colorimetric method for measuring total oxidant status. Clin. Biochem. 38, 1103-1111.

Georlette, D., Blaise, V., Collins, T., D’Amico, S., Gratia, E., Hoyoux, A., Marx, J.C., Sonan, G., Feller, G., Gerday, C., 2004. Some like it cold: biocatalysis at low temperatures. FEMS Microbiol. Rev. 28, 25-42.

Hanna, N., Ahmed, K., Anwar, M., Petrova, A., Hiatt, M., Hegyi, T., 2004. Effect of storage on breast milk antioxidant activity. Arch. Dis. Child. Fetal Neonatal Ed. 89, F51820.

Henriksen, L.O., Faber, N.R., Moller, M.F., Nexo, E., Hansen, A.B., 2014. Stability of 35 biochemical and immunological routine tests after 10 hours storage and transport of human whole blood at $21^{\circ} \mathrm{C}$. Scand. J. Clin. Lab. Invest. 74, 603-610.

Hooser, S.B., McCarthy, J.M., Wilson, C.R., Harms, J.L., Stevenson, G., Everson, R.J., 2000. Effects of storage conditions and hemolysis on vitamin E concentrations in porcine serum and liver. J. Vet. Diagn. Invest. 12, 365-368.

Jansen, E.H.J.M., Beekhof, P.K., Cremers, J.W.J.M., Viezeliene, D., Muzakova, V., Skalicky, J., 2013. Long-term stability of parameters of antioxidant status in human serum. Free Radic. Res. 47, 535-540.

Karlsen, A., Blomhoff, R., Gundersen, T.E., 2007. Stability of whole blood and plasma ascorbic acid. Eur. J. Clin. Nutr. 61, 1233-1236.

Key, T., Oakes, S., Davey, G., Moore, J., Edmond, L.M., McLoone, U.J., Thurnham, D.I., 1996. Stability of vitamins A, C, and E, carotenoids, lipids, and testosterone in whole 
blood stored at 4 degrees $\mathrm{C}$ for 6 and 24 hours before separation of serum and plasma. Cancer Epidemiol. Biomarkers Prev. 5, 811-814.

Koziorowska-Gilun, M., Koziorowski, M., Fraser, L., Strzezek, J., 2011. Antioxidant defence system of boar cauda epididymidal spermatozoa and reproductive tract fluids. Reprod. Domest. Anim. 46, 527-533.

Kumaresan, A., Kadirvel, G., Bujarbaruah, K.M., Bardoloi, R.K., Das, A., Kumar, S., Naskar, S., 2009. Preservation of boar semen at 18 degrees $\mathrm{C}$ induces lipid peroxidation and apoptosis like changes in spermatozoa. Anim. Reprod. Sci. 110, 162-171.

Li, J., Barranco, I., Tvarijonaviciute, A., Molina, M.F., Martinez, E.A., RodriguezMartinez, H., Parrilla, I., Roca, J., 2018. Seminal plasma antioxidants are directly involved in boar sperm cryotolerance. Theriogenology 107, 27-35.

Mahfouz, R., Sharma, R., Sharma, D., Sabanegh, E., Agarwal, A., 2009. Diagnostic value of the total antioxidant capacity (TAC) in human seminal plasma. Fertil. Steril. 91, 805-811.

Mathews-Roth, M.M., Stampfer, M.J., 1984. Some factors affecting determination of carotenoids in serum. Clin. Chem. 30, 459-461.

Oddoze, C., Lombard, E., Portugal, H., 2012. Stability study of 81 analytes in human whole blood, in serum and in plasma. Clin. Biochem. 45, 464-469.

Otasevic, V., Kalezic, A., Macanovic, B., Jankovic, A., Stancic, A., Garalejic, E., Korac, A., Korac, B., 2019. Evaluation of the antioxidative enzymes in the seminal plasma of infertile men: Contribution to classic semen quality analysis. Syst. Biol. Reprod. Med. 65, $343-349$.

Parrilla, I., del Olmo, D., Sijses, L., Martinez-Alborcia, M.J., Cuello, C., Vazquez, J.M., Martinez, E.A., Roca, J., 2012. Differences in the ability of spermatozoa from 
individual boar ejaculates to withstand different semen-processing techniques. Anim. Reprod. Sci. 132, 66-73.

Peeker, R., Abramsson, L., Marklund, S.L., 1997. Superoxide dismutase isoenzymes in human seminal plasma and spermatozoa. Mol. Hum. Reprod. 3, 1061-1066.

Roca, J., Broekhuijse, M.L.W.J., Parrilla, I., Rodriguez-Martinez, H., Martinez, E.A., Bolarin, A., 2015. Boar differences in artificial insemination outcomes: can they be minimized? Reprod. Domest. Anim. 50 Suppl 2, 48-55.

Roca, J., Parrilla, I., Bolarin, A., Martinez, E.A., Rodriguez-Martinez, H., 2016a. Will AI in pigs become more efficient? Theriogenology 86, 187-193.

Roca, J., Parrilla, I., Gil, M.A., Cuello, C., Martinez, E.A., Rodriguez-Martinez, H., 2016b. Non-viable sperm in the ejaculate: Lethal escorts for contemporary viable sperm. Anim. Reprod. Sci. 169, 24-31.

Rodriguez-Martinez, H., Kvist, U., Ernerudh, J., Sanz, L., Calvete, J.J., 201 1. Seminal plasma proteins: what role do they play? Am. J. Reprod. Immunol. 66 Suppl 1, 11-22.

Rossi, G., Giordano, A., Pezzia, F., Kjelgaard-Hansen, M., Paltrinieri, S., 2013. Serum paraoxonase 1 activity in dogs: preanalytical and analytical factors and correlation with C-reactive protein and alpha-2-globulin. Vet. Clin. Pathol. 42, 329-341.

Rubio, C.P., Tvarijonaviciute, A., Caldin, M., Hernández-Ruiz, J., Cerón, J.J., Martínez-Subiela, S., Tecles, F., 2018. Stability of biomarkers of oxidative stress in canine serum. Res. Vet. Sci. 121, 85-93.

Strzezek, J., Lapkiewicz, S., Lecewicz, M., 1999. A note on antioxidant capacity of boar seminal plasma. Anim. Sci. Pap. Reports 17, 181-188. 
Tanner, M., Kent, N., Smith, B., Fletcher, S., Lewer, M., 2008. Stability of common biochemical analytes in serum gel tubes subjected to various storage temperatures and times pre-centrifugation. Ann. Clin. Biochem. 45, 375-379.

Waberski, D., Riesenbeck, A., Schulze, M., Weitze, K.F., Johnson, L., 2019. Application of preserved boar semen for artificial insemination: Past, present and future challenges. Theriogenology 137, 2-7.

Wu, R., Feng, J., Yang, Y., Dai, C., Lu, A., Li, J., Liao, Y., Xiang, M., Huang, Q., Wang, D., Du, X.-B., 2017. Significance of serum total oxidant/antioxidant status in patients with colorectal cancer. PLoS One 12, e0170003.

Zura Zaja, I., Samardzija, M., Vince, S., Vilic, M., Majic-Balic, I., Duricic, D., Milinkovic-Tur, S., 2016. Differences in seminal plasma and spermatozoa antioxidative systems and seminal plasma lipid and protein levels among boar breeds and hybrid genetic traits. Anim. Reprod. Sci. 170, 75-82. 